\title{
Proof of a conjecture of Amdeberhan and Moll on a divisibility property of binomial coefficients
}

\author{
Quan-Hui Yang* \\ School of Mathematics and Statistics \\ Nanjing University of Information Science and Technology \\ Nanjing, P.R. China \\ yangquanhui010163.com
}

Submitted: Feb 10, 2014; Accepted: Dec 21, 2014; Published: Jan 9, 2015

Mathematics Subject Classifications: 05A10, 11B65

\begin{abstract}
Let $a, b$ and $n$ be positive integers with $a>b$. In this note, we prove that

$$
(2 b n+1)(2 b n+3)\left(\begin{array}{c}
2 b n \\
b n
\end{array}\right) \mid 3(a-b)(3 a-b)\left(\begin{array}{c}
2 a n \\
a n
\end{array}\right)\left(\begin{array}{c}
a n \\
b n
\end{array}\right) .
$$
\end{abstract}

This confirms a recent conjecture of Amdeberhan and Moll.

Keywords: binomial coefficients; $p$-adic order; divisibility properties

\section{Introduction}

In 2009, Bober [1] determined all cases such that

$$
\frac{\left(a_{1} n\right) ! \cdots\left(a_{k} n\right) !}{\left(b_{1} n\right) ! \cdots\left(b_{k+1} n\right) !} \in \mathbb{Z}
$$

where $a_{s} \neq b_{t}$ for all $s, t, \sum a_{s}=\sum b_{t}$ and $\operatorname{gcd}\left(a_{1}, \ldots, a_{k}, b_{1}, \ldots, b_{k+1}\right)=1$.

Recently, Z.-W. Sun $[12,13]$ studied divisibility properties of binomial coefficients and obtained some interesting results. For example,

$$
2(2 n+1)\left(\begin{array}{c}
2 n \\
n
\end{array}\right) \mid\left(\begin{array}{c}
6 n \\
3 n
\end{array}\right)\left(\begin{array}{c}
3 n \\
n
\end{array}\right),
$$

*Supported by the National Natural Science Foundation of China, Grant No. 11371195 and the Startup Foundation for Introducing Talent of NUIST, Grant No. 2014r029. 


$$
(10 n+1)\left(\begin{array}{c}
3 n \\
n
\end{array}\right) \mid\left(\begin{array}{c}
15 n \\
5 n
\end{array}\right)\left(\begin{array}{c}
5 n-1 \\
n-1
\end{array}\right)
$$

Later, Guo and Krattenthaler (see [5, 8]) obtained some similar divisibility results. Related results appear in [2]-[4] and [7]-[11].

Introduce the notation

$$
S_{n}=\frac{\left(\begin{array}{c}
6 n \\
3 n
\end{array}\right)\left(\begin{array}{c}
3 n \\
n
\end{array}\right)}{2(2 n+1)\left(\begin{array}{c}
2 n \\
n
\end{array}\right)} \quad \text { and } \quad t_{n}=\frac{\left(\begin{array}{c}
15 n \\
5 n
\end{array}\right)\left(\begin{array}{c}
5 n-1 \\
n-1
\end{array}\right)}{(10 n+1)\left(\begin{array}{c}
3 n \\
n
\end{array}\right)}
$$

In [6], Guo proved the conjectures due to Z.W. Sun $[12,13]$.

Theorem A. ([12, Conjecture 3(i)].) Let $n$ be a positive integer. Then

$$
3 S_{n} \equiv 0 \quad(\bmod 2 n+3) .
$$

Theorem B. ([13, Conjecture 1.3].) Let $n$ be a positive integer. Then

$$
21 t_{n} \equiv 0 \quad(\bmod 10 n+3)
$$

Recently, T. Amdeberhan and V. H. Moll proposed a conjecture related to Theorems A and B, which was only presented as Conjecture 7.1 in Guo's paper [6] by private communication.

This notes provides a proof of this conjecture.

Theorem 1. Let $a, b$ and $n$ be positive integers with $a>b$. Then

$$
(2 b n+1)(2 b n+3)\left(\begin{array}{c}
2 b n \\
b n
\end{array}\right) \mid 3(a-b)(3 a-b)\left(\begin{array}{c}
2 a n \\
a n
\end{array}\right)\left(\begin{array}{c}
a n \\
b n
\end{array}\right) .
$$

Remark 2. Theorem A is the special case $a=3, b=1$ of Theorem 1 .

\section{Proofs}

For a real number $z$, denote the greatest integer not exceeding $z$ by $\lfloor z\rfloor$ and $\{z\}$ denotes the fractional part of $z$. For an integer $n$ and a prime $p$, write $p^{k} \| n$ if $p^{k} \mid n$ and $p^{k+1} \nmid n$. The integer $k$ above is denoted by $\nu_{p}(n)$.

It is well known that

$$
\nu_{p}(n !)=\sum_{i=1}^{\infty}\left\lfloor\frac{n}{p^{i}}\right\rfloor .
$$

The proof of Theorem 1 begins with a preliminary result.

Lemma 3. Let $x$ and $y$ be two real numbers. Then

$$
\lfloor 2 x\rfloor+\lfloor y\rfloor \geqslant\lfloor x\rfloor+\lfloor x-y\rfloor+\lfloor 2 y\rfloor .
$$


Proof. The identity $2 x+y=x+(x-y)+2 y$ shows that it suffices to $\{2 x\}+\{y\} \leqslant$ $\{x\}+\{x-y\}+\{2 y\}$. The proof now follows by comparing $\{x\}$ and $\{y\}$ to $1 / 2$. The details are left to the reader.

Proof of Theorem 1. Let

$$
T(a, b, n):=\left(\begin{array}{c}
2 a n \\
a n
\end{array}\right)\left(\begin{array}{c}
a n \\
b n
\end{array}\right) /\left(\begin{array}{c}
2 b n \\
b n
\end{array}\right)=\frac{(2 a n) !(b n) !}{(a n) !(a n-b n) !(2 b n) !} .
$$

By (1), for any prime $p$,

$$
\nu_{p}(T(a, b, n))=\sum_{i=1}^{\infty}\left(\left\lfloor\frac{2 a n}{p^{i}}\right\rfloor+\left\lfloor\frac{b n}{p^{i}}\right\rfloor-\left\lfloor\frac{a n}{p^{i}}\right\rfloor-\left\lfloor\frac{a n-b n}{p^{i}}\right\rfloor-\left\lfloor\frac{2 b n}{p^{i}}\right\rfloor\right) .
$$

Lemma 3 shows that each term of $\nu_{p}(T(a, b, n))$ is nonnegative. Hence $\nu_{p}(T(a, b, n)) \geqslant 0$. Therefore, $T(a, b, n) \in \mathbb{Z}$.

Since $\operatorname{gcd}(2 b n+1,2 b n+3)=1$, it suffices to prove that

$$
2 b n+1 \mid 3(a-b)(3 a-b) T(a, b, n)
$$

and

$$
2 b n+3 \mid 3(a-b)(3 a-b) T(a, b, n) .
$$

The second statement is established here. The proof of the first statement is similar and the details are omitted. Suppose that $p^{\alpha} \| 2 b n+3$ with $\alpha \geqslant 1$. It is shown that

$$
p^{\alpha} \mid 3(a-b)(3 a-b) T(a, b, n) .
$$

Let $p^{\beta} \| a-b$ and $p^{\gamma} \| 3 a-b$ with $\beta \geqslant 0$ and $\gamma \geqslant 0$. Write $\tau=\max \{\beta, \gamma\}$. If $\alpha \leqslant \tau$, then (2) clearly holds. Now we assume $\alpha>\tau$.

Suppose that $p \geqslant 5$. The statement

$$
\left\lfloor\frac{2 a n}{p^{i}}\right\rfloor+\left\lfloor\frac{b n}{p^{i}}\right\rfloor-\left\lfloor\frac{a n}{p^{i}}\right\rfloor-\left\lfloor\frac{a n-b n}{p^{i}}\right\rfloor-\left\lfloor\frac{2 b n}{p^{i}}\right\rfloor=1
$$

is established for $i=\tau+1, \tau+2, \ldots, \alpha$. This is proven next. Noting that $p \mid 2 b n+3$ and $p \geqslant 5$, it follows that $\operatorname{gcd}(p, n)=1$.

Observe that $p^{\alpha} \| 2 b n+3$, it follows that $2 b n \equiv p^{\alpha}-3\left(\bmod p^{\alpha}\right)$ and $b n \equiv\left(p^{\alpha}-3\right) / 2$ $\left(\bmod p^{\alpha}\right)$.

Take $i \in\{\tau+1, \tau+2, \ldots, \alpha\}$. Then $2 b n \equiv p^{i}-3\left(\bmod p^{i}\right)$ and $b n \equiv\left(p^{i}-3\right) / 2$ $\left(\bmod p^{i}\right)$. Now we divide into several cases according to the value of an $\left(\bmod p^{i}\right)$.

Case 1. an $\equiv t\left(\bmod p^{i}\right)$ with $0 \leqslant t<\left(p^{i}-3\right) / 2$. It follows that $2 a n \equiv 2 t\left(\bmod p^{i}\right)$ and $0 \leqslant 2 t<p^{i}-3$. Also

$$
a n-b n \equiv t-\left(p^{i}-3\right) / 2+p^{i} \quad\left(\bmod p^{i}\right),
$$


where $0 \leqslant t-\left(p^{i}-3\right) / 2+p^{i}<p^{i}$. Hence

$$
\begin{aligned}
& \left\lfloor\frac{2 a n}{p^{i}}\right\rfloor+\left\lfloor\frac{b n}{p^{i}}\right\rfloor-\left\lfloor\frac{a n}{p^{i}}\right\rfloor-\left\lfloor\frac{a n-b n}{p^{i}}\right\rfloor-\left\lfloor\frac{2 b n}{p^{i}}\right\rfloor \\
= & \frac{2 a n-2 t}{p^{i}}+\frac{b n-\left(p^{i}-3\right) / 2}{p^{i}}-\frac{a n-t}{p^{i}} \\
& -\left(\frac{a n-b n-\left(t-\left(p^{i}-3\right) / 2+p^{i}\right)}{p^{i}}\right)-\frac{2 b n-\left(p^{i}-3\right)}{p^{i}} \\
= & 1 .
\end{aligned}
$$

Case 2. an $\equiv\left(p^{i}-3\right) / 2\left(\bmod p^{i}\right)$. Then, $a n-b n \equiv 0\left(\bmod p^{i}\right) . \operatorname{Since} \operatorname{gcd}(p, n)=1$, it follows that $p^{i} \mid a-b$. However, $p^{\beta} \| a-b$ and $\beta \leqslant \tau<i$. This is a contradiction.

Case 3. an $\equiv\left(p^{i}-1\right) / 2\left(\bmod p^{i}\right)$. It follows that

$$
3 a n-b n \equiv \frac{3\left(p^{i}-1\right)}{2}-\frac{\left(p^{i}-3\right)}{2} \equiv 0 \quad\left(\bmod p^{i}\right) .
$$

The fact that $\operatorname{gcd}(p, n)=1$ implies $p^{i} \mid 3 a-b$. This contradicts $p^{\gamma} \| 3 a-b$ and $\gamma<i$.

Case 4. an $\equiv t\left(\bmod p^{i}\right)$ with $\left(p^{i}+1\right) / 2 \leqslant t<p^{i}$. Then

$$
2 a n \equiv 2 t-p^{i} \quad\left(\bmod p^{i}\right), \quad 0 \leqslant 2 t-p^{i}<p^{i},
$$

and

$$
a n-b n \equiv t-\left(p^{i}-3\right) / 2 \quad\left(\bmod p^{i}\right), \quad 0 \leqslant t-\left(p^{i}-3\right) / 2<p^{i} .
$$

Hence

$$
\begin{aligned}
& \left\lfloor\frac{2 a n}{p^{i}}\right\rfloor+\left\lfloor\frac{b n}{p^{i}}\right\rfloor-\left\lfloor\frac{a n}{p^{i}}\right\rfloor-\left\lfloor\frac{a n-b n}{p^{i}}\right\rfloor-\left\lfloor\frac{2 b n}{p^{i}}\right\rfloor \\
= & \frac{2 a n-\left(2 t-p^{i}\right)}{p^{i}}+\frac{b n-\left(p^{i}-3\right) / 2}{p^{i}}-\frac{a n-t}{p^{i}} \\
& -\left(\frac{a n-b n-\left(t-\left(p^{i}-3\right) / 2\right)}{p^{i}}\right)-\frac{2 b n-\left(p^{i}-3\right)}{p^{i}} \\
= & 1 .
\end{aligned}
$$

Therefore, $\nu_{p}(T(a, b, n)) \geqslant \alpha-\tau$, and this implies

$$
\nu_{p}(3(a-b)(3 a-b) T(a, b, n)) \geqslant \alpha .
$$

The proof of $(2)$, for $p \geqslant 5$, is complete.

Now assume $p=3$. If $9 \mid n$, then $3 \mid 2 b n+3$ and $9 \nmid 2 b n+3$. It follows that $\alpha=1$, and then (2) clearly holds. If $9 \nmid n$, then the proof of the case $p \geqslant 5$ applies to this situation. In Case 2 , an $-b n \equiv 0\left(\bmod 3^{i}\right)$ gives $3^{i-1} \mid a-b$. In Case $3,3^{i-1} \mid 3 a-b$. Thus, if $i \geqslant \tau+2$, then $i-1 \geqslant \tau+1$. It is a contradiction in both cases. Hence

$$
\left\lfloor\frac{2 a n}{3^{i}}\right\rfloor+\left\lfloor\frac{b n}{3^{i}}\right\rfloor-\left\lfloor\frac{a n}{3^{i}}\right\rfloor-\left\lfloor\frac{a n-b n}{3^{i}}\right\rfloor-\left\lfloor\frac{2 b n}{3^{i}}\right\rfloor=1
$$


for $i=\tau+2, \tau+3, \ldots, \alpha$. It follows that $\nu_{3}(T(a, b, n)) \geqslant \alpha-\tau-1$, and then

$$
\nu_{3}(3(a-b)(3 a-b) T(a, b, n)) \geqslant \alpha .
$$

That is, (2) also holds. Hence, $2 b n+3 \mid 3(a-b)(3 a-b) T(a, b, n)$.

Therefore,

$$
(2 b n+1)(2 b n+3)\left(\begin{array}{c}
2 b n \\
b n
\end{array}\right) \mid 3(a-b)(3 a-b)\left(\begin{array}{c}
2 a n \\
a n
\end{array}\right)\left(\begin{array}{c}
a n \\
b n
\end{array}\right) .
$$

This completes the proof of Theorem 1.

\section{Acknowledgements}

We are grateful to the anonymous referee for carefully reading our original manuscript and giving many detailed comments.

\section{References}

[1] J. W. Bober. Factorial ratios, hypergeometric series, and a family of step functions. J. Lond. Math. Soc., 79:422-444, 2009.

[2] N. J. Calkin. Factors of sums of powers of binomial coefficients. Acta Arith., 86:17-26, 1998.

[3] H.-Q. Cao, H. Pan. Factors of alternating binomial sums. J. Adv. in Appl. Math., 45:96-107, 2010.

[4] N. J. Fine. Binomial coefficients modulo a prime. Amer. Math. Monthly, 54:589-592, 1947.

[5] V. J. W. Guo. Proof of Sun's conjecture on the divisibility of certain binomial sums. Electron. J. Combin., 20(4):\#P4.20, 2013.

[6] V. J. W. Guo. Proof of two divisibility properties of binomial coefficients conjectured by Z.-W. Sun. Electron. J. Combin., 21(2):\#P2.54, 2014.

[7] V. J. W. Guo, F. Jouhet and J. Zeng, Factors of alternating sums of products of binomial and q-binomial coefficients. Acta Arith., 127:17-31, 2007.

[8] V. J. W. Guo, C. Krattenthaler. Some divisibility properties of binomial and qbinomial coefficients. J. Number Theory, 135:167-184, 2014.

[9] V. J. W. Guo, J. Zeng. Factors of binomial sums from the Catalan triangle. J. Number Theory, 130:172-186, 2010.

[10] V. J. W. Guo, J. Zeng. Factors of sums and alternating sums involving binomial coefficients and powers of integers. Int. J. Number Theory, 7:1959-1976, 2011.

[11] M. Razpet. On divisibility of binomial coefficients. Discrete Math., 135:377-379, 1994. 
[12] Z.-W. Sun. On divisibility of binomial coefficients. J. Austral. Math. Soc., 93:189-201, 2012.

[13] Z.-W. Sun. Products and sums divisible by central binomial coefficients. Electron. J. Combin., 20(1):\#P1.9, 2013. 